\title{
Improvement in muscle strength with low-load isotonic training depends on fascicle length but not joint angle
}

\section{$\operatorname{AUTHOR}(\mathrm{S})$ :}

Tanaka, Hiroki; Ikezoe, Tome; Nakamura, Masatoshi; Yanase, Ko; Fujita, Kosuke; Motomura, Yoshiki; Kusano, Ken; ... Saeki, Junya; Morishita, Katsuyuki; Ichihashi, Noriaki

\section{CITATION:}

Tanaka, Hiroki ... [et al]. Improvement in muscle strength with low-load isotonic training depends on fascicle length but not joint angle. Muscle \& Nerve 2018, 57(1): $83-89$

\section{ISSUE DATE:}

2018-01

\section{URL:}

http://hdl.handle.net/2433/231298

\section{RIGHT:}

This is the accepted version of the following article: [Muscle \& Nerve, Volume57, Issuel, January 2018, Pages 83-89], which has been published in final form at https://doi.org/10.1002/mus.25601. This article may be used for noncommercial purposes in accordance with Wiley Terms and Conditions for Self-Archiving.; The full-text file will be made open to the public on 08 December 2018 in accordance with publisher's 'Terms and Conditions for Self-Archiving'.; This is not the published version. Please cite only the published version.; この論文は出版社版でありません。引用の際には 出版社版をご確認じ利用ください。 
1 Title : Improvement in muscle strength with low-load isotonic training depends on fascicle length but

2 not joint angle

3

4 Authors: Hiroki Tanaka ${ }^{1 *}$, M.Sc., Tome Ikezoe ${ }^{2}$, Ph.D., Masatoshi Nakamura ${ }^{3}$, Ph.D., Ko Yanase²,

5 M.Sc., Kosuke Fujita ${ }^{4}$, M.Sc., Yoshiki Motomura² ${ }^{2}$ B.Sc., Ken Kusano², B.Sc., Kojiro Araki ${ }^{5}$, M.Sc.,

6 Jun Umehara², M.Sc., Junya Saeki², M.Sc., Katsuyuki Morishita², Ph.D., Noriaki Ichihashi², Ph.D.

7 1) Rehabilitation Unit, Kyoto University Hospital, 54, Shogoin-Kawahara-cho, Sakyo-ku, Kyoto 606-8507, Japan.

9 2) Human Health Sciences, Graduate School of Medicine, Kyoto University, Kyoto, Japan

3) Institute for Human Movement and Medical Sciences, Niigata University of Health and Welfare, Niigata, Japan

4) Rehabilitation Group, Department of Medical Technique, Nagoya University Hospital, Nagoya, Japan

5) Department of Rehabilitation, Sapporo Tokushukai Hospital, Hokkaido, Japan

Acknowledgements: We are grateful for the contributions of the undergraduate and graduate research assistants in the Kyoto University for their assistance in performing the experiments. There was no funding for this project.

19 Conflict of Interest: NONE

20 Financial Disclosure: NONE

Running title: Specific adaptation of low-load training

Word count: 142 words (abstract), 3803 words (not including the abstract, abbreviations, and references)

Ethical Publication Statement: We confirm that we have read the Journal's position on issues involved in ethical publication and affirm that this report is consistent with those guidelines.

\section{*Corresponding author:}

Hiroki Tanaka. R.P.T., M.Sc.

Rehabilitation Unit, Kyoto University Hospital 54 Shogoin-Kawahara-cho, Sakyo-ku, Kyoto 606-8507, Japan.

E-mail: tanaka.hiroki.8w@kyoto-u.ac.jp 


\section{ABSTRACT}

2 Introduction: This study investigates whether low-load isotonic training will elicit greater

3 improvement in muscle strength at the same fascicle length, rather than at the same joint angle.

4 Methods: 16 healthy men (24.1 \pm 2.5 years) were randomly divided into intervention and control

5 groups. The pre- and post-training, maximum isometric and isokinetic strengths, and the fascicle

6 length of medial gastrocnemius muscle were measured. Isotonic resistance training at $15^{\circ}$ to $30^{\circ}$ ankle

7 plantar flexion at low intensity was conducted for 4 weeks. Results: The maximum isometric and

8 isokinetic strength of the intervention group increased significantly only at $15^{\circ}$ dorsiflexion and $8^{\circ}$ to

$912^{\circ}$ dorsiflexion. Fascicle length during maximum voluntary contraction at $15^{\circ}$ to $0^{\circ}$ dorsiflexion was

10 similar to fascicle length under training conditions. Discussion: It is possible that the improvement in

11 muscle strength with low-load training depends on fascicle length rather than joint angle.

13 Key words: Plantar flexion; Ultrasound; Triceps surae; Specificity of training; Training adaptation,

14 Low-load 
isometric training, Kitai and Sale (1989) ${ }^{1}$ reported that ankle plantar flexion training using maximum of the foot) produces an improvement in the maximum isometric strength at an ankle position of only around $0^{\circ}$ (between $-5^{\circ}$ and $5^{\circ}$ ). These results demonstrate the so-called joint angle specificity on training effect ${ }^{1}$. Moreover, it was reported that the muscle strength specifically improves at the training angle used during not only isometric training but also isotonic and isokinetic training. Graves et al. (1989) $)^{2}$ and Barak et al. (2004) $)^{3}$ respectively examined the effect of isotonic and isokinetic highload training of knee extension, and reported that the improvement in maximum isometric strength occurred at the training angle. On the other hand, some reports have demonstrated that an improvement in maximum muscle strength can be obtained over a wide range of joint angles around the training angle during isometric training ${ }^{4-7}$ or eccentric isokinetic training ${ }^{3}$. Thus, because a consensus view regarding joint angle specificity has yet to be achieved, further research and investigation are required. contraction is needed to gain an improvement in strength during resistance training ${ }^{8}$. Therefore, all

17 previous studies investigating the joint angle specificity of the training effect used a high load of more than $70 \%$ maximum voluntary isometric contraction or $60 \%$ one repetition maximum (1RM). 
1

2

3

4

5

However, our recent study ${ }^{9}$ showed that isometric training with a low-intensity $30 \%$ maximum voluntary contraction was effective for improving the muscle strength at a different joint angle from the training angle. Our findings suggest that the effects of low-intensity training depend on not the joint angle specificity but on the fascicle length. The fascicle length is affected by both the joint angle and the contraction level. During high-load training, because the forces exerted during training are similar to those during the measurement of maximum isometric strength, the fascicle length during training will also be similar at the same joint angle used when measuring maximum strength. This suggests that it is uncertain whether the training effects depend on the joint angle or the fascicle length during high-load training. From the perspective of our previous study ${ }^{9}$, during isotonic training, it can also be perceived that an improvement in muscle strength is affected by fascicle length, which is determined from the load and joint angle. However, the influence of the fascicle length on the improvement in muscle strength during low-load isotonic training is unclear.

The purpose of this study is to examine whether an improvement in muscle strength depends solely on the joint angle, or rather on the fascicle length, which is affected by the training load and joint angle, when applying low-load isotonic plantar flexion training. We hypothesized that low-load isotonic training will result in a greater improvement in muscle strength at the same fascicle length, rather than at the same joint angle used during training. 
Participants

16 healthy men (24.1 \pm 2.5 years in age), non-athletes with no involvement in regular

stretching or resistance training, participated in this study. Persons with a history of neuromuscular

5 attended three familiarization sessions, practicing maximum voluntary isometric, isokinetic, and

Procedures

in Figure 1. Prior to the pre-training, all subjects were familiarized with the maximum voluntary contraction procedure for plantar flexion. During the initial week of the experiment, the subjects isotonic contractions. For the maximum voluntary isometric contractions, the subjects practiced the four ankle joint positions used for testing, set at $15^{\circ}$ intervals over a range from $15^{\circ}$ dorsiflexion to 
$130^{\circ}$ plantar flexion. For the maximum voluntary isokinetic contractions, the subjects practiced at $20^{\circ} / \mathrm{s}$

2 over a range from $15^{\circ}$ dorsiflexion to $30^{\circ}$ plantar flexion. For the maximum voluntary isotonic

3 contractions (1RM), the subjects practiced three ranges of ankle joint angle ( $15^{\circ}$ dorsiflexion to $0^{\circ}, 0^{\circ}$

4 to $15^{\circ}$ plantar flexion, and $15^{\circ}$ plantar flexion to $30^{\circ}$ plantar flexion). An ankle joint angle of $0^{\circ}$ (neutral

5 position) was defined as a $90^{\circ}$ angle between the fibula and fifth metatarsal bone. Following the

6 familiarization sessions, the muscle strength during maximum voluntary contraction of the ankle

7 plantar flexors, the fascicle length, pennation angle, and muscle thickness were measured pre-training.

8 The same measurements were conducted after 4 weeks of training, resulting in a total experimental

9 period of five weeks.

The intervention group underwent isotonic resistance training of ankle plantar flexion at an

intensity of 20\% 1RM, 3 days per week for 4 weeks, using a Biodex dynamometer (Biodex System 4,

14 Biodex Medical Systems, Inc., Shirley, New York, USA.). The isotonic resistance training protocol

consisted of 3 sets of 20 repetitions of plantar flexion contraction of only the concentric phase,

16 performed at $15^{\circ}$ to $30^{\circ}$ plantar flexion, with a 1-s rest between each contraction, and a 2-min rest

17 between sets. Isotonic training was conducted at a speed of $5 \%$ s over an ankle angle range of $15^{\circ}$ to

$1830^{\circ}$ resulting in a 3-s movement per repetition. The subjects were instructed to maintain a fixed 
1 inserted between the velcro strap and the instep to prevent any unwanted movement of the ankle joint.

velocity on the dynamometer using a metronome. An examiner supervised all training sessions and checked whether the subjects were able to exert force at the proper speed. The subjects in the control group did not receive any intervention.

\section{Procedure of muscle strength measurement}

The muscle strength of each subject was determined by measuring the maximum voluntary isometric strength, maximum isokinetic voluntary isokinetic strength, and 1RM using a Biodex dynamometer with a sampling rate of $1000 \mathrm{~Hz}$. For each measurement, the ankle joint of the dominant leg was securely attached to the footplate of the dynamometer using a velcro strap. A soft cloth was

11 The trunk and distal thigh were securely fixed using dynamometer belts to keep the hip joint position at an $80^{\circ}$ flexion and the knee joint at full extension. The subjects grasped the horizontal bars attached to the dynamometer. Before each test, the subjects underwent a warm up of 10 to 20 submaximal

14 isometric contractions. After more than $1 \mathrm{~min}$ of rest, the subjects were asked to generate their maximum voluntary isometric and isokinetic contractions in random order. The measurement of their 1RM was obtained last because it caused significant fatigue. Total measurement time was about 60 $\min$. 
1 order of maximum voluntary isometric contraction randomized across the participants. Their

2 maximum voluntary isometric contraction was exerted for $5 \mathrm{~s}$ at each of the four ankle joint angles,

3 with more than 2 min of rest provided between each maximum voluntary isometric contraction. Their

4 maximum isometric strength was measured twice at each ankle joint angle, and the greater value of

5 the two measures was used for the analysis.

72 min of rest between trials. Their isokinetic strength was measured at every $1^{\circ}$ across the range of

$815^{\circ}$ dorsiflexion to $30^{\circ}$ plantar flexion. Their maximum isokinetic strength was determined to have

9 the highest value of isokinetic strength. The greater value of two measurements was used for the analysis. Moreover, their isokinetic strength measured every $1^{\circ}$ across the range of $15^{\circ}$ dorsiflexion to $30^{\circ}$ plantar flexion was also used for analysis. subject was unable to apply the required full range of plantarflex motion, at which time the load was as 1RM. A rest period was allotted between each attempt to ensure recovery. 
2 at proximal $30 \%$ of the lower leg length ${ }^{10}$, using B-mode ultrasound imaging (LOGIQ e, General

3 Electric, Duluth, GA, USA) with an 8-MHz linear array probe $(6 \mathrm{~cm})$ with a gain of 58 to $70 \mathrm{~dB}$. The

4 depth and dynamic focus settings of the equipment were controlled to achieve a clear image of the

5 MG. The fascicle length was measured under isotonic training conditions (at $15^{\circ}$ to $30^{\circ}$ plantar flexion

6 with 20\% 1RM) and during muscle strength measurements pre- and post-training. In the measurement

7 of ultrasound imaging at maximum isometric strength, the images were preserved when the exerted

8 force displayed on the dynamometer monitor reached a plateau. The images during isokinetic strength

9 measurements and under isotonic training conditions were measured using the moving imaging function. A static sonographic image was gained at each $2^{\circ}$ of plantar flexion because the speed of the dynamometer was $20^{\circ} / \mathrm{s}$ and the image was recorded every $0.1 \mathrm{~s}$.

2. The fascicle length was estimated from these images based on the methods used to evaluate the distance along a straight line, between the extension lines from the aponeurosis, and the origin of the fascicle as previously described for the quadriceps muscle ${ }^{11-14}$. The reliability of this method for the measurements of the MG was shown in our previous study ${ }^{9}$. The intraclass correlation coefficient

17 (ICC 1.1) for the fascicle length of the MG was showed good reliability for both the inter session (ICC

$18>0.9)$ and inter day $(\text { ICC }>0.75)^{11}$. The pennation angle was defined as an angle of the fascicle and 
1

deep aponeurosis. The fascicle length and pennation angle were measured under each condition using image processing software (ImageJ, version 1.48, National Institutes of Health, Bethesda, MD, USA). To examine the morphological changes, the fascicle length, the pennation angle, and the muscle thickness of the MG, lateral gastrocnemius (LG), and soleus (SOL) at rest with an ankle position of $0^{\circ}$ were measured pre- and post-training in the proximal $30 \%$ of the lower leg. The muscle thickness was measured to assess whether the improvement in muscle strength was due to muscle hypertrophy, by measuring the line drawn perpendicular from the surface to the deep aponeurosis along the transverse plane. To accurately measure the muscle thickness without including noncontractile tissue, measurements between the inside edges of the aponeurosis were used. Previous studies showed reliability of the ultrasound technique for measuring the muscle thickness of the triceps surae $^{12,13}$.

\section{Statistical analysis}

Statistical analysis was conducted using SPSS (version 22.0, IBM Japan Inc., Tokyo, Japan).

The normality of the data was evaluated using a Shapiro-Wilk test. Group differences regarding the characteristics and maximum muscle strength at the baseline were assessed using an unpaired t-test.

A split-plot ANOVA with two factors (group $\times$ time) was used to analyze the effects on the maximum muscle strength, the fascicle length, the pennation angle, and muscle thickness. A two-way 
1

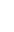

repeated measures ANOVA with two factors (ankle joint angle $\times$ time) was used to determine the differences in isokinetic strength. A paired t-test was used to determine significant differences between the value pre- and post-training when significant interactions or main effects were found.

The paired t-test was used to determine the differences in fascicle length between isotonic training conditions (at $15^{\circ}$ to $30^{\circ}$ plantar flexion with $20 \% 1 \mathrm{RM}$ ) and during muscle strength measurements at each angle in the intervention group in post-training. The fascicle length under the training conditions was calculated as the mean value at $15^{\circ}$ to $30^{\circ}$ plantar flexion in pre- and posttraining. The differences were considered to be statistically significant at an alpha level of 0.05. RESULTS

None of the subjects dropped out, and all subjects in the intervention group completed the training sessions. There were no significant differences in age, height, or body mass between the intervention group ( $23.0 \pm 1.1$ years, $174.0 \pm 7.0 \mathrm{~cm}, 70.3 \pm 8.2 \mathrm{~kg}$ ) and control group ( $25.2 \pm 3.0$ years, $171.1 \pm 3.4 \mathrm{~cm}, 63.0 \pm 6.8 \mathrm{~kg}$ ). There were also no significant differences in any variables of their maximum muscle strength at the baseline between the two groups.

Effects of isotonic training intervention on maximum muscle strength 
2 at any ankle angle. Main effects for time were significant at $15^{\circ}$ dorsiflexion $(\mathrm{p}=0.02)$, but were not significant at $0^{\circ}, 15^{\circ}$, and $30^{\circ}$ plantar flexion. At $15^{\circ}$ dorsiflexion, the maximum isometric strength post-training was significantly higher than pre-training in the intervention group $(\mathrm{p}=0.03)$, but there was no significant change in the control group.

The split-plot ANOVA, using two factors (group $\times$ time), showed no significant interactions or a main effect of maximum isokinetic strength and 1RM at any range of ankle angle between preand post-training in both groups.

The changes in isokinetic strength over a range of ankle angles from $15^{\circ}$ dorsiflexion to $30^{\circ}$ plantar flexion are shown in Figure 3. Two-way repeated measures ANOVA (ankle joint angle $\times$ time) showed a significant interaction in the intervention group. There were significant increases in isokinetic strength at $12^{\circ}$ to $8^{\circ}$ dorsiflexion. However, there were no significant differences in the control group.

Differences in fascicle length between under isotonic training conditions and during muscle strength measurements

In the intervention group, the mean value of the fascicle length under the training conditions was $3.54 \pm 0.64 \mathrm{~cm}$. 

contraction at $15^{\circ}$ and $30^{\circ}$ plantar flexion. However, there were no significant differences between the training conditions and maximum isometric contraction at $15^{\circ}$ dorsiflexion and at $0^{\circ}$. ankle angle from $15^{\circ}$ dorsiflexion to $30^{\circ}$ plantar flexion is showed in Figure 4 . There were no significant differences in the fascicle length between the training conditions and the isokinetic strength measurements between $5^{\circ}$ and $1^{\circ}$ dorsiflexion.

Effects of intervention on fascicle length, pennation angle, and muscle thickness an ankle position of $0^{\circ}$ are shown in Table 2. No significant differences were found in fascicle length, pennation angle, or muscle thickness between pre- and post-training in either group. Moreover, no significant differences were found in fascicle length or pennation angle during maximum muscle strength measurement between pre- and post-training (see Table 3). 


\section{DISCUSSION}

to $30^{\circ}$ plantar flexion resulted in a significant improvement in maximum isometric strength at $15^{\circ}$ dorsiflexion, and isokinetic strength at $8^{\circ}$ to $12^{\circ}$ dorsiflexion.

Our results show that there was a significant improvement in maximum isometric strength at $15^{\circ}$ dorsiflexion in only the intervention group, though they received isotonic training at ankle angles of $15^{\circ}$ to $30^{\circ}$ plantar flexion. That is, there was no significant change in isometric strength at the training angle. This result is inconsistent with the joint angle specificity of the training effect as proposed for isometric ${ }^{1}$ and isotonic ${ }^{2}$ training. Our previous study ${ }^{9}$ reported that the improvement of muscle strength in isometric training depended on fascicle length rather than joint angle. In this study, there was no significant difference in fascicle length during low-load training at $15^{\circ}$ to $30^{\circ}$ plantar flexion $(3.54 \pm 0.64 \mathrm{~cm})$ and the measurement of maximum isometric strength at $15^{\circ}$ dorsiflexion (3.71 $\pm 1.03 \mathrm{~cm}$ ). This result implies that the fascicle length during training was similar to that of maximum voluntary isometric contraction regardless of the different ankle angles applied. Our findings suggest that low-load isotonic training may be effective for improving the maximum isometric strength not at the same joint angle but at the same fascicle length during training, and that the effects of low-load isotonic training with a shortened fascicle length can be produced at a more 
1

lengthened fascicle position.

Our results also show that isokinetic strength improved significantly only within the range of $12^{\circ}$ to $8^{\circ}$ dorsiflexion, without a significant increase in maximum isokinetic strength. This result suggests that there was an improvement in muscle strength specifically within the range of $12^{\circ}$ to $8^{\circ}$ dorsiflexion, which was not influenced from the improvement of the maximum voluntary isokinetic contraction. Similar to the result of the maximum isometric strength, the result of the isokinetic strength was also inconsistent with the joint angle specificity of the training effect. The fascicle length at $5^{\circ}$ to $0^{\circ}$ dorsiflexion of the maximum isokinetic contraction was almost the same as the fascicle length during training. Unfortunately, the results of our study are inconsistent with our hypothesis that an improvement in muscle strength depends on the fascicle length during training, because the isokinetic strength improved within the range of $12^{\circ}$ to $8^{\circ}$ dorsiflexion. The discrepancy may be due to a lack of perfect synchronization between a dynamometer and ultrasound imaging. In this study, the initial movement of the dynamometer and the initial muscle contraction in the ultrasound imaging were synchronized manually. If the muscle contraction occurs before the movement of dynamometer, the data on the fascicle length in the ultrasound imaging and the joint angle do not match. An inadequate synchronization might be the reason for the lack of improvement in isokinetic strength at the same fascicle length. Further investigation of the fascicle length through proper synchronization between the dynamometer and ultrasound imaging is needed. 
2 reflects the weakest strength within each angle range. Generally, plantar flexion strength decreases

3 with shortened fascicle length, which is seen at an angle with greater plantar flexion. Therefore, it is

4 possible that the $1 \mathrm{RM}$ of the ankle angle from $15^{\circ}$ dorsiflexion to $0^{\circ}$, from $0^{\circ}$ to $15^{\circ}$ plantar flexion,

5 and from $15^{\circ}$ to $30^{\circ}$ plantar flexion were influenced by the maximum muscle strength at $0^{\circ}, 15^{\circ}$, and

$630^{\circ}$ plantar flexion, respectively. In fact, the present study shows that there were no significant

7 improvements in maximum isometric strength at $0^{\circ}, 15^{\circ}$, and $30^{\circ}$ plantar flexion, although there was

8 a significant improvement in the maximum isometric strength at $15^{\circ}$ dorsiflexion. Although it is

9 speculative, the absence of improvement in isometric strength at $0^{\circ}, 15^{\circ}$, and $30^{\circ}$ plantar flexion may explain the reason for the absence in improvement of $1 \mathrm{RM}$ from $15^{\circ}$ dorsiflexion to $0^{\circ}$, from $0^{\circ}$ to $15^{\circ}$ plantar flexion, and from $15^{\circ}$ to $30^{\circ}$ plantar flexion.

In this study, there were no significant differences in the fascicle length, the pennation angle, or the muscle thickness between pre- and post-training, suggestingthat the improvement in muscle strength was not caused by morphological changes but by neural adaptations. However, we could not measure the moment arm of triceps surae muscles. It was assumed that the moment arm of triceps surae muscles would be longer if muscle hypertrophy occurred because the moment arm becomes

17 longer by the muscle bulge when the plantarflexor muscles contract ${ }^{14}$. In this study, it was assumed that the moment arm of triceps surae did not change because muscle thickness did not change after the 
1

training. The measurement of moment arm of triceps surae could be explored in the future.

As a limitation of this study, we did not investigate the effects of neural adaptation.

Improvement in muscle strength after resistance training depends on neural adaptations over the initial period of training ( $<4$ weeks), followed by morphological muscle adaptations after 6 to 8 weeks, which mainly contribute to the strength gains ${ }^{15,16}$. Our results show no changes in morphological measurements such as the fascicle length, the pennation angle, and the muscle thickness after training.

Therefore, the improvement in maximum muscle strength after the 4-week intervention applied in this study may be influenced by neural adaptations, such as increases in the muscle activity of the agonist muscles and decreases in antagonist co-activation ${ }^{17}$. However, we did not measure the muscle activity because it may not be appropriate to compare muscle activity during maximum strength measurements between pre- and post- training, which were normalized with respect to the maximum voluntary contraction measured on different days. Further investigation is necessary to clarify the interactions between the neural adaptation mechanisms and the effects of specificity in the fascicle length during training. A second limitation of this study was the small sample size, which might cause an unclear interpretation of the relationship between the maximum isometric strength and the fascicle length. In fact, with regard to the changes in the maximum isometric strength at $0^{\circ}$ of dorsiflexion, there was no significant improvement after training. Moreover, with regard to isokinetic strength, our hypothesis was also not clearly established because of inadequate synchronization between the dynamometer and 
1 ultrasound measurements. Therefore, our hypothesis of the specificity of fascicle length may not be

2 supported completely in this study. The last limitation was that our study considered the fascicle length

3 only for the MG because the reliability of the fascicle length measurements at various ankle joint

4 angles and contraction levels is only certain for the MG muscle ${ }^{9}$. Therefore, the influence of other

5 plantar flexor muscles such as SOL and LG on improvements in muscle strength is unclear.

7 fascicle length improves isometric and isokinetic strength at different angles from angles used during

8 training. The results suggest that the effects of the improvement in muscle strength may depend on

9 fascicle length rather than joint angle during isotonic training. Thus, low-load training with a shortened

11 position. It is possible that low-load training at a shortened muscle length may be more suitable and joint motions.

15 Abbreviations:

$16 \quad 1 \mathrm{RM}-$ one repetition maximum

$17 \quad$ MG - medial gastrocnemius

18 ICC - intraclass correlation coefficient 
$1 \mathrm{SOL}$-soleus

2 LG - lateral gastrocnemius

$3 \quad$ SD - standard deviation

$4 \quad$ DF-dorsiflexion

$5 \quad \mathrm{PF}-$ plantar flexion 
3 1. Kitai TA, Sale DG. Specificity of joint angle in isometric training. Eur J Appl Physiol 4 Occup Physiol 1989;58(7):744-748.

52 Graves JE, Pollock ML, Jones AE, Colvin AB, Leggett SH. Specificity of limited 6 range of motion variable resistance training. Med Sci Sports Exerc 1989;21(1):84-89.

$7 \quad 3 . \quad$ Barak Y, Ayalon M, Dvir Z. Transferability of strength gains from limited to full 8 range of motion. Med Sci Sports Exerc 2004;36(8):1413-1420.

9 4. Rasch PJ, Pierson WR. One position versus multiple positions in isometric exercise. 10 Am J Phys Med 1964;43:10-12.

11 5. Rasch PJ, Pierson WR, Logan GA. The effect of isometric exercise upon the strength 12 of antagonistic muscles. Int Z Angew Physiol 1961;19:18-22.

136 Weir JP, Housh TJ, Weir LL. Electromyographic evaluation of joint angle specificity 14 and cross-training after isometric training. J Appl Physiol 1994;77(1):197-201.

157 Weir JP, Housh TJ, Weir LL, Johnson GO. Effects of unilateral isometric strength 16 training on joint angle specificity and cross-training. Eur J Appl Physiol Occup Physiol $17 \quad$ 1995;70(4):337-343.

18 8. Ratamess NA, Alvar BA, Evetoch TE, Housh TJ, Ben Kibler W, Kraemer WJ, 19 Triplett NT. American College of Sports Medicine position stand. Progression Models in 20 Resistance Training for Healthy Adults. Med Sci Sports Exerc 2009;41(3):687-708.

219 Tanaka H, Ikezoe T, Umehara J, Nakamura M, Umegaki H, Kobayashi T, Nishishita 22 S, Fujita K, Araki K, Ichihashi N. Influences of Fascicle Length During Isometric Training 23 on Improvement of Muscle Strength. J Strength Cond Res 2016;30(11):3249-3255.

24 10. Akagi R, Takahashi H. Effect of a 5-week static stretching program on hardness of 25 the gastrocnemius muscle. Scand J Med Sci Sports 2014;24(6):950-957.

26 11. Lee J, Koh D, Ong CN. Statistical evaluation of agreement between two methods 27 for measuring a quantitative variable. Comput Biol Med 1989;19(1):61-70.

28 12. Maganaris CN, Baltzopoulos V, Sargeant AJ. In vivo measurements of the triceps 29 surae complex architecture in man: implications for muscle function. J Physiol 1998;512 ( Pt 30 2):603-614.

31 13. Narici MV, Binzoni T, Hiltbrand E, Fasel J, Terrier F, Cerretelli P. In vivo human 32 gastrocnemius architecture with changing joint angle at rest and during graded isometric 33 contraction. J Physiol 1996;496 ( Pt 1):287-297.

34 14. Maganaris CN, Baltzopoulos V, Sargeant AJ. Changes in Achilles tendon moment 
1 arm from rest to maximum isometric plantarflexion: in vivo observations in man. J Physiol $2 \quad 1998 ; 510($ Pt 3):977-985.

3 15. Kraemer WJ, Fleck SJ, Evans WJ. Strength and power training: physiological 4 mechanisms of adaptation. Exerc Sport Sci Rev 1996;24:363-397.

5 16. Moritani T, Devries HA. Neural factors versus hypertrophy in the time course of 6 muscle strength gain. J Phys Med Rehabil 1979;58(3):115-130.

7 17. Garfinkel S, Cafarelli E. Relative changes in maximal force, EMG, and muscle cross8 sectional area after isometric training. Med Sci Sports Exerc 1992;24(11):1220-1227.

9 
2 Table 1 Effects of intervention on maximum muscle strength (Nm)

\begin{tabular}{|c|c|c|c|c|c|c|c|c|c|c|}
\hline & \multicolumn{4}{|c|}{ intervention group $(\mathrm{n}=\mathbf{8})$} & \multicolumn{4}{|c|}{ control group $(n=8)$} & \multicolumn{2}{|c|}{ F value } \\
\hline & \multicolumn{2}{|c|}{ Pre-training } & \multicolumn{2}{|c|}{ Post-training } & \multicolumn{2}{|c|}{ Pre-training } & \multicolumn{2}{|c|}{ Post-training } & \multirow[t]{2}{*}{ Interaction } & \multirow[t]{2}{*}{ Main effect } \\
\hline Maximum ison & strength & & & & & & & & & \\
\hline DF15 $^{\circ}$ & 195.11 & \pm 42.92 & 206.89 & $\pm 36.60 *$ & 207.31 & \pm 52.07 & 219.53 & \pm 47.36 & $\mathrm{~F}<0.1$ & $\mathrm{~F}=7.48^{*}$ \\
\hline $0^{\circ}$ & 167.24 & \pm 30.25 & 176.35 & \pm 28.02 & 177.65 & \pm 45.78 & 174.81 & \pm 38.44 & $\mathrm{~F}=2.12$ & $\mathrm{~F}=0.59$ \\
\hline PF $15^{\circ}$ & 127.29 & \pm 26.49 & 126.51 & \pm 23.22 & 123.60 & \pm 33.58 & 120.39 & \pm 30.23 & $\mathrm{~F}=0.22$ & $F=0.60$ \\
\hline PF $30^{\circ}$ & 78.53 & \pm 21.37 & 79.48 & \pm 24.05 & 75.54 & \pm 28.80 & 72.84 & \pm 25.13 & $\mathrm{~F}=0.90$ & $\mathrm{~F}=0.21$ \\
\hline \multicolumn{11}{|c|}{ Maximum isokinetic strength } \\
\hline & 140.16 & \pm 20.35 & 146.43 & \pm 26.76 & 161.16 & \pm 45.81 & 160.96 & \pm 42.36 & $F=0.55$ & $F=0.49$ \\
\hline \multicolumn{11}{|l|}{ 1RM } \\
\hline $\mathrm{DF}^{\circ} 5^{\circ}$ to $0^{\circ}$ & 189.38 & \pm 33.85 & 195.00 & \pm 23.45 & 191.25 & \pm 38.98 & 192.50 & \pm 34.95 & $\mathrm{~F}=0.87$ & $\mathrm{~F}=0.17$ \\
\hline $0^{\circ}$ to $\mathrm{PF} 15^{\circ}$ & 146.88 & \pm 31.50 & 151.25 & \pm 31.14 & 146.88 & \pm 31.05 & 146.25 & \pm 33.25 & $F=0.18$ & $F=0.31$ \\
\hline $\mathrm{PF}^{\circ} 5^{\circ}$ to $30^{\circ}$ & 103.13 & \pm 27.64 & 100.00 & \pm 22.99 & 99.38 & \pm 26.52 & 98.13 & \pm 28.90 & $F=0.46$ & $F=0.10$ \\
\hline
\end{tabular}

$3 \quad$ Values are expressed as mean $\pm \mathrm{SD}$ (standard deviation)

$4 \quad \mathrm{DF}=$ dorsiflexion, $\mathrm{PF}=$ plantar flexion

$5 \quad$ F value are showed for interaction (group $\times$ time) and main effect (time) in sprit plot ANOVA.

$6 \quad *$ significant difference between pre- and post-training $(P<0.05)$ 
1 Table 2 Effects of intervention on fascicle length, muscle thickness, and pennation angle

\begin{tabular}{|c|c|c|c|c|c|c|c|c|c|c|c|c|c|c|c|c|c|c|c|}
\hline \multirow[b]{3}{*}{ MG } & \multirow[b]{3}{*}{ IG } & \multicolumn{6}{|c|}{ Fascicle length $(\mathrm{cm})$} & \multicolumn{6}{|c|}{ Pennation angle $\left(^{\circ}\right)$} & \multicolumn{6}{|c|}{ Muscle thickness（cm） } \\
\hline & & \multicolumn{3}{|c|}{ Pre-training } & \multicolumn{3}{|c|}{ Post-training } & \multicolumn{3}{|c|}{ Pre-training } & \multicolumn{3}{|c|}{ Post-training } & \multicolumn{3}{|c|}{ Pre-training } & \multicolumn{3}{|c|}{ Post-training } \\
\hline & & 5.93 & \pm & 1.29 & 6.26 & \pm & 1.08 & 24.39 & \pm & 5.45 & 21.45 & \pm & 1.61 & 2.25 & \pm & 0.82 & 2.02 & \pm & 0.34 \\
\hline & CG & 6.31 & \pm & 1.35 & 6.49 & \pm & 1.45 & 22.84 & \pm & 2.99 & 23.77 & \pm & 1.97 & 2.13 & \pm & 0.45 & 2.12 & \pm & 0.61 \\
\hline \multirow[t]{2}{*}{ LG } & IG & 7.29 & \pm & 1.75 & 6.81 & \pm & 1.77 & 17.92 & \pm & 1.78 & 18.62 & \pm & 3.99 & 1.67 & \pm & 0.24 & 1.63 & \pm & 0.38 \\
\hline & CG & 7.84 & \pm & 1.88 & 7.92 & \pm & 2.46 & 16.94 & \pm & 3.40 & 18.99 & \pm & 3.77 & 1.70 & \pm & 0.44 & 1.78 & \pm & 0.41 \\
\hline \multirow[t]{2}{*}{ SOL } & IG & 4.49 & \pm & 0.86 & 4.21 & \pm & 1.13 & 20.16 & \pm & 1.47 & 20.31 & \pm & 5.61 & 1.69 & \pm & 0.57 & 1.69 & \pm & 0.46 \\
\hline & CG & 5.06 & \pm & 1.90 & 4.97 & \pm & 1.27 & 19.75 & \pm & 7.84 & 20.66 & \pm & 5.55 & 1.73 & \pm & 0.50 & 1.80 & \pm & 0.52 \\
\hline
\end{tabular}

2 Values are expressed as mean \pm SD (standard deviation)

$3 \quad \mathrm{MG}=$ medial gastrocnemius, $\mathrm{LG}=$ lateral gastrocnemius, $\mathrm{SOL}=$ soleus

4 IG = intervention group, $\mathrm{CG}=$ control group 


\begin{tabular}{|c|c|c|c|c|c|c|c|c|c|c|}
\hline & \multicolumn{5}{|c|}{ Fascicle length $(\mathrm{cm})$} & \multicolumn{5}{|c|}{ Pennation angle $\left({ }^{\circ}\right)$} \\
\hline & \multicolumn{2}{|c|}{ intervention group $(\mathrm{n}=\mathrm{8})$} & \multicolumn{3}{|c|}{ control group $(n=8)$} & \multicolumn{2}{|c|}{ intervention group $(\mathrm{n}=8)$} & \multicolumn{3}{|c|}{ control group $(n=8)$} \\
\hline & Pre-training & Post-training & Pre-1 & training & Post-training & Pre-training & Post-training & Pre- & training & Post-training \\
\hline $\mathbf{0}^{\circ}$ & \pm 0.93 & $3.18 \pm 0.82$ & 3.23 & \pm 1.04 & $3.09 \pm 0.73$ & \pm 9.43 & \pm 8.38 & 37.98 & \pm 9.23 & $41.48 \pm 8.69$ \\
\hline PF $15^{\circ}$ & $2.72 \pm 0.77$ & $2.72 \pm 0.67$ & 2.79 & \pm 0.69 & $2.93 \pm 0.69$ & \pm 9.24 & \pm 6.43 & 42.77 & \pm 8.09 & $42.68 \pm 7.49$ \\
\hline
\end{tabular}

2 Values are expressed as mean $\pm \mathrm{SD}$ (standard deviation) 
1 Fig. 1 Flowchart of the present study.

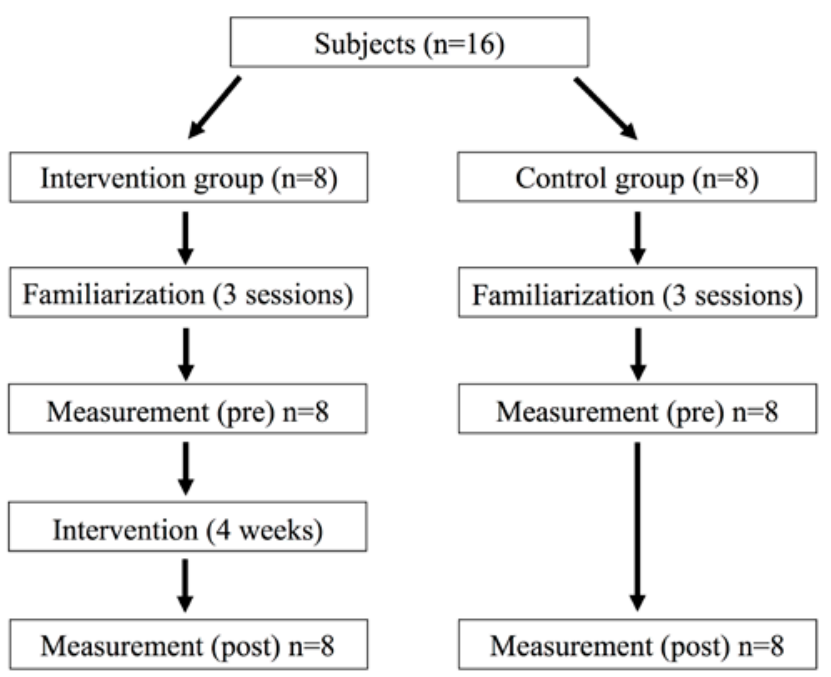

2

3 pre $=$ pre-training, post $=$ post-training 
1 Fig. 2 Estimation of fascicle length.

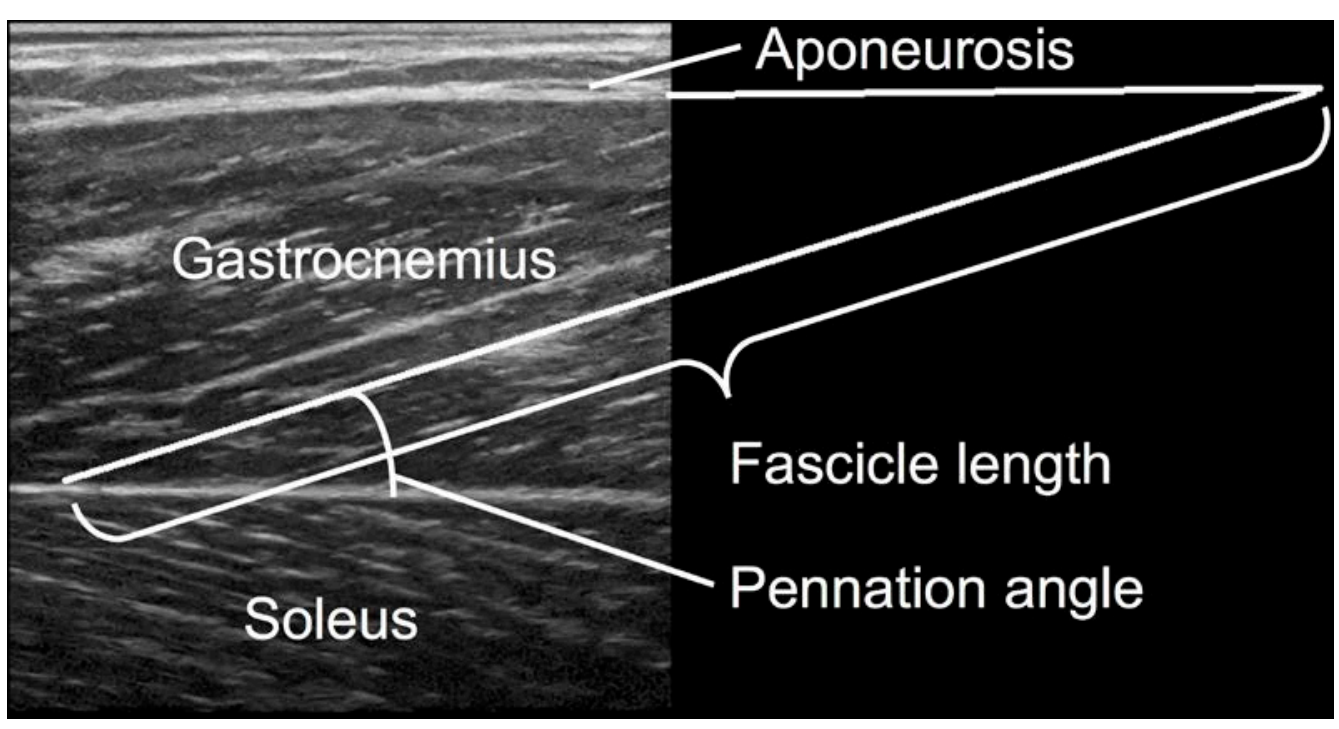

3 The fascicle length of the medial gastrocnemius muscle was defined as the distance in a straight line

4 between the extension lines from the aponeurosis and the fascicle origin. 
1 Fig. 3 Change in isokinetic strength at each ankle angle between pre- and post-training.
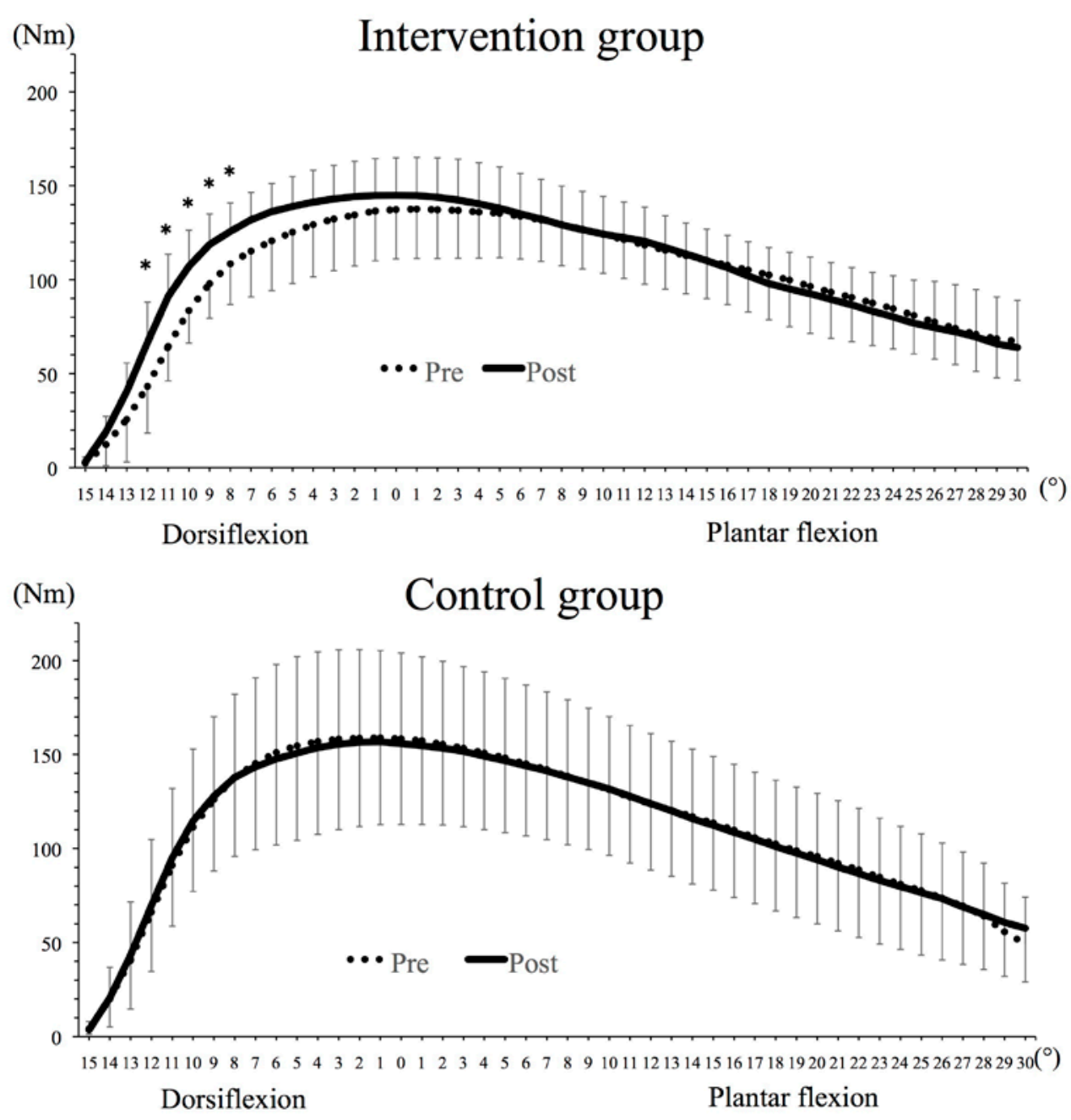

$3 *$ indicates significant difference between pre- and post-training $(\mathrm{P}<0.05)$. 
1 Fig. 4 Differences in fascicle length between isotonic training conditions and during maximum

2 isokinetic strength measurements.

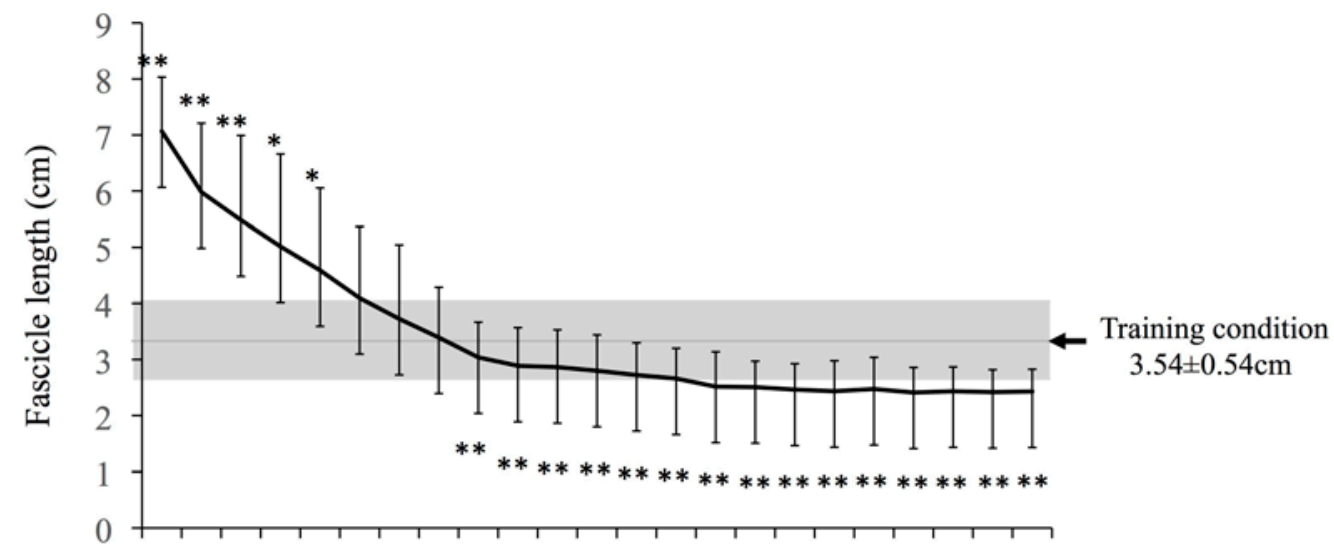

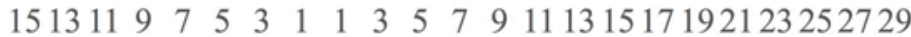

Dorsiflexion

Plantar flexion

3

Joint angle $\left({ }^{\circ}\right)$

$4 *$ indicates significant difference from the fascicle length under training condition $\left({ }^{* *} P<0.01\right.$,

$5 \quad * P<0.05)$.

6 The line indicated by the arrowlabeled "Training condition" shows the mean value of the fascicle

7 length during isotonic training. The gray area shows the standard deviation of the value of the fascicle

8 length during training. 